\title{
Erfahrungen aus dem Einsatz von Pflegerobotern für Menschen im Alter
}

\author{
Michael Früh und Alina Gasser
}

\begin{abstract}
Zusammenfassung
Dieser Beitrag beschreibt die Erfahrungen bei der Entwicklung, Erprobung und Kommerzialisierung von Pflegerobotern aus Sicht der Firma F\&P Robotics AG. Vorbehalte gegenüber dem Einsatz von Robotik in der Pflege sind noch immer weit verbreitet. Die Haltung von F\&P ist es, dass nicht das Ersetzen von Mitarbeitern, sondern die sinnvolle Zusammenarbeit von Mensch und Roboter gefördert werden soll. F\&P ist ein Pionier in den Bereichen Personal- und Pflegerobotik und testet derzeit den Einsatz von Robotern in Alten- und Pflegeheimen. Im ersten Teil des Beitrags werden verschiedene Serviceroboter von F\&P beschrieben und deren Einsatzbereiche und Nutzen erläutert. Im Rahmen einer Masterarbeit der Universität Basel wurde eine Studie bezüglich der Akzeptanz älterer Menschen gegenüber Pflegerobotern durchgeführt. Die Resultate werden im zweiten Teil präsentiert.
\end{abstract}

M. Früh $(\square)$

F\&P Robotics AG, Glattbrugg, Schweiz

E-Mail: mfr@fp-robotics.com

A. Gasser

Zürich, Schweiz

E-Mail: ags@fp-robotics.com

(C) Der/die Autor(en) 2018

O. Bendel (Hrsg.), Pflegeroboter, https://doi.org/10.1007/978-3-658-22698-5_3 


\subsection{F\&P Robotics}

F\&P Robotics ist ein Hightech-Unternehmen mit Sitz in Zürich. Der Schwerpunkt der Firma liegt auf der Entwicklung von intelligenten, persönlichen und sicheren Robotern. Dabei wird Wert auf eine sympathische Erscheinung der Roboter gelegt, um die Beziehung von Mensch und Maschine möglichst angenehm zu gestalten.

Die Produkte von F\&P kommen in zwei Märkten zum Einsatz. Der erste Markt umfasst das Gesundheitswesen mit Schwerpunkt Pflege, wobei F\&P intelligente Unterstützungsroboter für Pflegeheime, Krankenhäuser und Rehabilitationseinrichtungen anbietet. Die beiden Roboter Lio und P-Care unterstützen das Pflegepersonal bei repetitiven Aufgaben und bieten den Bewohnern Assistenz- und Unterhaltungsmöglichkeiten. Der zweite Markt umfasst das Gewerbe. Für Kunden in diesem Segment bietet F\&P sichere und einfach zu bedienende Roboterarme an. Der Roboterarm P-Rob unterstützt Mitarbeiter in direkter Zusammenarbeit beim Beladen von Maschinen, beim Verpacken von Produkten oder bei der Qualitätskontrolle. Die Roboter können dabei stationär, aber auch auf fahrbaren Plattformen eingesetzt werden.

\subsection{Pflegeroboter und deren Funktionen}

Die Konversationen rund um das Thema Pflegerobotik sind in den letzten Jahren merklich häufiger geworden. Dies liegt einerseits daran, dass das Gesundheitswesen mit Herausforderungen konfrontiert ist, für die dringend innovative Lösungen benötigt werden. Dazu zählen die demografische Entwicklung und der Mangel an Fachkräften. Andererseits werden von Robotikherstellern und Universitäten vermehrt Projekte vorgestellt, welche einen Nutzen für die Praxis vorweisen können.

Die aktuellen Funktionalitäten von Pflegerobotern werden ihrem Namen allerdings nur selten gerecht. Interaktionsfunktionen, wie Terminerinnerungen oder Spiele, stehen im Vordergrund. Gewisse Roboter können auch Transportfunktionen übernehmen oder als Gehilfe dienen. Um Aufgaben der Pflege bewältigen zu können, beispielsweise einen Verband zu wechseln oder Blut zu nehmen, benötigen Roboter aus technischer Sicht ein komplexes Zusammenspiel zwischen physischen Manipulationen, Sicherheitsmechanismen und Wahrnehmungsfähigkeit. Aus ethischer und sozialer Sicht stellen sich weitere wichtige Fragen, welchen sich die Autoren dieses Buches ebenfalls widmen.

In diesem Spannungsfeld hat die F\&P Robotics AG in enger Zusammenarbeit mit Partnern aus dem Gesundheitswesen Pflegeroboter entwickelt, welche gemäß Klein et al. (2018) als komplexe Systeme bezeichnet werden können und Funktionen in der Betreuung, Hotellerie und der Pflegeunterstützung abdecken. Dies aus der Überzeugung heraus, dass nur durch umfangreiche Funktionalitäten ein effektiver Mehrwert für die Praxis generiert werden kann, der zudem auch finanziell vertretbar ist.

Die Roboter von F\&P zeichnen sich dadurch aus, dass sie für unterschiedliche Einsatzbereiche und Bedürfnisse angepasst werden können. So werden keine statischen 
Produkte entwickelt, sondern persönliche Serviceroboter, die auf Menschen eingehen und lernfähig sind. Dies macht sie besonders sinnvoll für die Pflege, wo sich die Bedürfnisse der Menschen laufend ändern können. Besonders in Alten- und Pflegeheimen fallen verschiedene Bereiche zusammen, da sie nicht nur auf Gesundheit ausgerichtet sind, sondern für viele Menschen deren Zuhause darstellen.

Ein wichtiger Bereich in der stationären Pflege stellt die Hotellerie dar. Serviceroboter können das Fachpersonal gezielt entlasten, indem sie Objekte - beispielsweise Gläser oder Teller - transportieren. Hierbei spielt die künstliche Intelligenz eine wichtige Rolle. Die Roboter müssen in der Lage sein, Gegenstände auch dann wiederzuerkennen, wenn sie nicht mehr an derselben Stelle liegen wie beim letzten Mal. Wie Klein et al. bemerken, sind besonders schwer erreichbare Gegenstände eine große Hürde für viele Patienten, welche durch die Hilfe von Robotern überwunden werden kann (2018, S. 67).

Auch in der Betreuung kommen die Serviceroboter zum Einsatz. Durch Interaktion und Unterhaltung bereiten die beiden Roboter Lio und P-Care, die im kommenden Abschnitt genauer vorgestellt werden, pflegebedürftigen Menschen Freude. Durch Sprachfunktionen können Unterhaltungen geführt und Informationen ausgetauscht werden. Lio und P-Care können Fragen nach dem Wetter oder den aktuellsten Nachrichten beantworten und dienen so als Gesprächspartner für die Bewohner. Sie können sich Wünsche und Verhaltensweisen ihres Gegenübers merken und so gezielt auf deren Bedürfnisse eingehen.

Nebst Hotellerie und Betreuung ist eine direkte Pflegeunterstützung durch Serviceroboter möglich. Lio kann sich mit Wearables verbinden, dadurch den Gesundheitszustand der Bewohner beobachten und dem Fachpersonal gezielt wichtige Informationen weiterleiten. Die Roboter können Patienten an Medikamente erinnern und, wenn z. B. nach einem Sturz notwendig, einen Alarm auslösen.

\subsubsection{Lio}

Lio ist ein persönlicher Serviceroboter für den Einsatz in Alten- und Pflegeheimen, Rehabilitationskliniken und für auf Unterstützung angewiesene Menschen zu Hause (s. Abb. 3.1). Der mobile, mit einer weichen Kunstlederhaut ausgestattete Roboter kann mit Menschen kommunizieren, im Haushalt und bei pflegerischen Aufgaben unterstützen. Technologien der Mechatronik und künstlichen Intelligenz werden so eingesetzt, dass Lio nicht nur hilfreich ist, sondern von Menschen auch gemocht und akzeptiert wird.

Komponenten von Lio:

- Roboterarm mit sechs Freiheitsgraden, max. Traglast $3 \mathrm{~kg}$

- Mobile Plattform mit Lasersensor und mehreren Ultraschallsensoren, autonome Navigation, Pfaderkennung (SLAM integriert), Ausweichfunktionen

- Ablageflächen für Getränke, Teller etc.

- Tablet-Display für Darstellung des Status von Lio an der Plattform 
Abb. 3.1 Roboter Lio

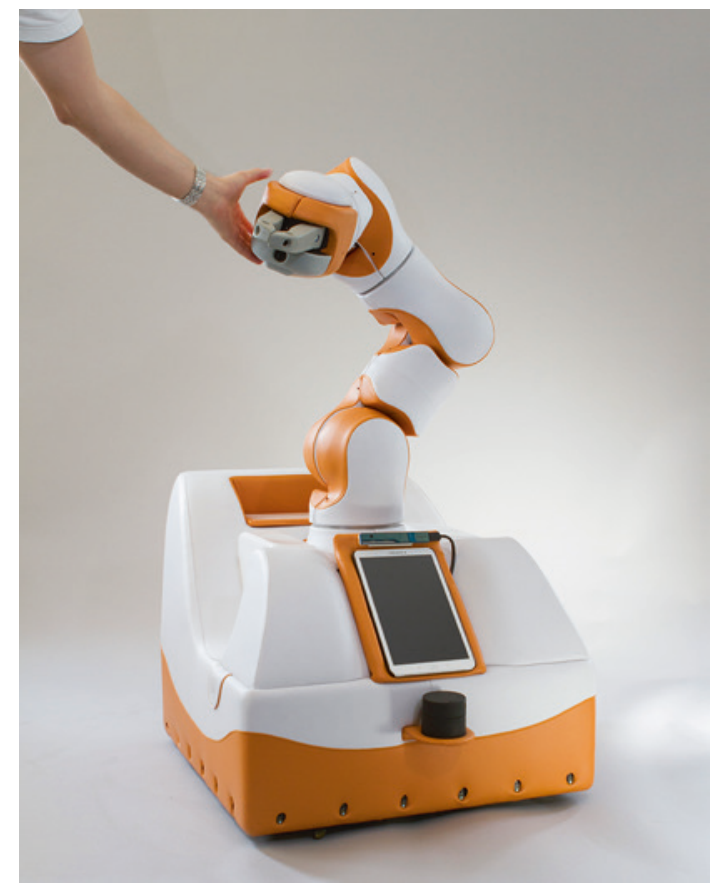

- Separates Bediener-Tablet

- Zwei Kameras für Personen- und Objekterkennung

- Softwarepaket mit ca. 30 Interaktionsfunktionen

Um Lio bestmöglich an die Bedürfnisse der Menschen anzupassen, wird sein Verhaltensrepertoire vor dem Einsatz auf die Menschen in seiner Umgebung eingestellt. Diese Anpassungen umfassen Spracherkennung, Personenerkennung, Tagesablauf, Usanzen der Person und spezielle Wünsche, sofern diese technisch realisierbar sind. Umfassendere Anpassungen (Funktionen, welche nicht im Standardrepertoire enthalten sind) werden projektspezifisch umgesetzt.

Ein Vorbehalt, der häufig im Zusammenhang mit Pflegerobotern geäußert wird, betrifft die Sicherheit der zu betreuenden Menschen. Die Sicherheit des Personals und der $\mathrm{zu}$ betreuenden Personen wird durch das Einhalten der entsprechenden Normen (insbesondere ISO 13482 Personal Care Robots und ISO 15066 Collaborative Robots) gewährleistet. Die Zulassung für Lio in Europa wird zusammen mit der Schweizerischen Unfallversicherungsanstalt (SUVA) bearbeitet. Zusätzlich wird das Personal, welches mit dem Roboter zusammenarbeitet, gezielt geschult. Die Schulung beinhaltet auch die Vermittlung von Sicherheitsmaßnahmen, welche das Pflegepersonal berücksichtigen soll.

Die Akzeptanz gegenüber Lio wurde bereits früh in der Entwicklungsphase mit Praxiseinsätzen und während einer Masterarbeit in Schweizer Altersheimen getestet (siehe 1.5). Lio half dabei, Gegenstände aufzuheben, und kommunizierte mit den Bewohnerinnen 
und Bewohnern. Die Reaktionen der älteren Menschen sind dabei mehrheitlich positiv ausgefallen. Zusätzlich werden Messen dazu genutzt, Erkenntnisse zum Einsatz von Pflegerobotern zu gewinnen. Dabei helfen Demos und Fragebogen, das Feedback von Besuchern festzuhalten und auszuwerten.

\subsubsection{P-Care}

P-Care wurde in Kooperation mit ZRFN Robotics aus China entwickelt (s. Abb. 3.2). Ähnlich wie Lio hilft P-Care Menschen mit Unterstützungsbedarf in ihrem Alltag und assistiert dem Pflegepersonal. P-Care zeichnet sich durch ein humanoides Design aus und vermittelt durch seine freundliche Erscheinung eine warme Atmosphäre. Er kann

Abb. 3.2 Roboter P-Care

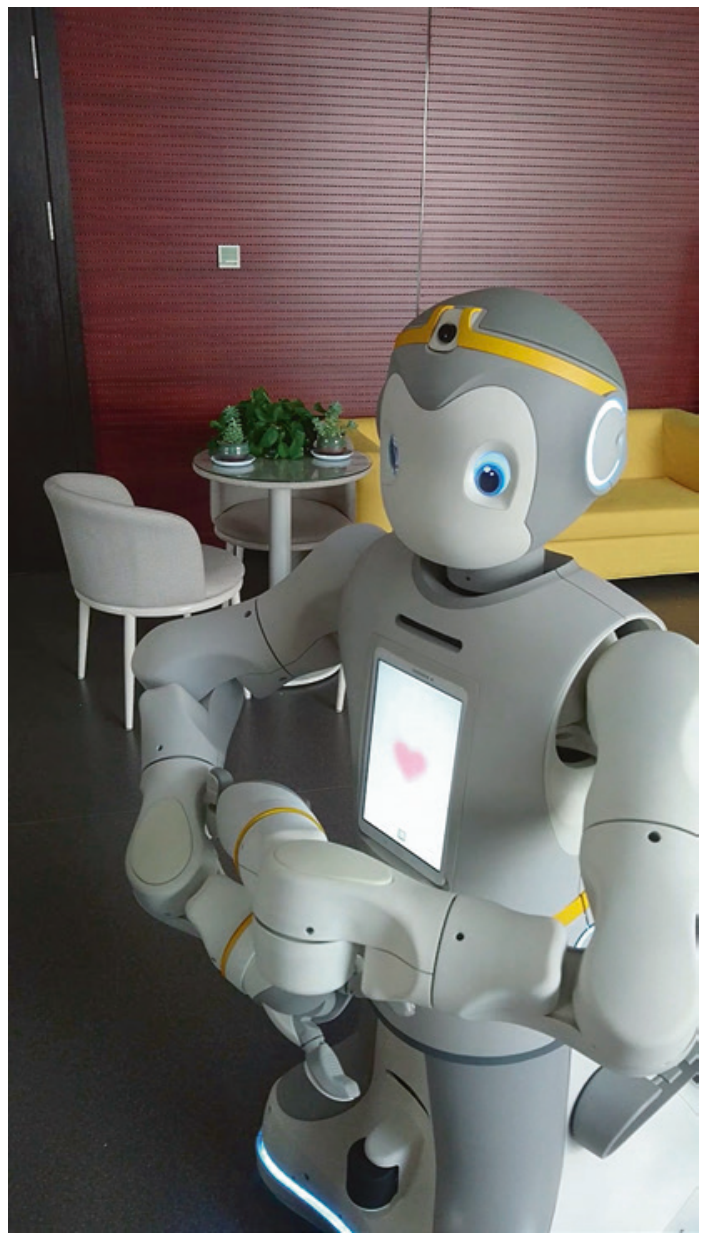


mit zwei Armen gleichzeitig arbeiten, hat ein integriertes Tablet zur Darstellung seines Zustandes sowie Mikrofon und Lautsprecher für die verbale Kommunikation.

Komponenten von P-Care:

- Roboter mit zwei Armen mit jeweils sechs Freiheitsgraden, max. Traglast je 3 kg

- Total 17 Freiheitsgrade, 5 davon im Rumpf des Roboters

- Dimensionen: ca. 1,5 m hoch, 0,6 m breit

- Mobile Plattform mit 30Ah, Lidarsensor, mehreren Time-of-Flight-(ToF)-Sensoren, autonomer Navigation, Pfaderkennung (SLAM), Ausweichfunktionen

- Ablage für Getränke, Teller etc.

- Tablet für Visualisierungen

- Emotionen können über LCD-Augen und integrierte LEDs ausgedrückt werden

- Zwei RGB-D-Kameras, vier RGB-Kameras

- Fortgeschrittene Softwarefunktionen: Spracherkennung (multilingual), soziale Funktionalitäten, integrierte künstliche Intelligenz

- Gesichtserkennung, Human-Pose-Erkennung

- Berührungsempfindliche Oberfläche am Kopf und Unterarm für haptische Interaktion

- Softwarepaket mit ca. 30 Interaktionsfunktionen

Mit über 30 Softwarefunktionen (Unterhaltung, Erinnerung an Medikamente oder Aktivitäten, soziale Interaktion etc.) kann P-Care auf individuelle Bedürfnisse der Menschen eingehen. Wie Lio kann P-Care in unterschiedlichen Bereichen wie Hotellerie, Betreuung und Pflegeunterstützung eingesetzt werden. Die persönliche Erscheinung ist aufgrund seines Designs bei P-Care besonders ausgeprägt. Das Design von P-Care ist im Gegensatz zu Lio menschenähnlich und vorerst auf den asiatischen Markt ausgerichtet.

\subsubsection{Therapieroboter P-Rob Glarus}

F\&P hat den Roboterarm P-Rob zu einem gefühlvollen Therapieroboter weiterentwickelt (s. Abb. 3.3). Dieser wird in der Tagesklinik Glarus in der Schweiz eingesetzt. Komponenten von P-Rob 2R:

- Sechs Freiheitsgrade

- Kraft-Momenten-Sensor

- Zwei Kameras

- Max. Traglast 3-5 kg

- Höhe: ca. $1,1 \mathrm{~m}$

- Reichweite: $77,5 \mathrm{~cm}$ 
Abb. 3.3 Therapieroboter P-Rob 2R in der Tagesklinik Glarus

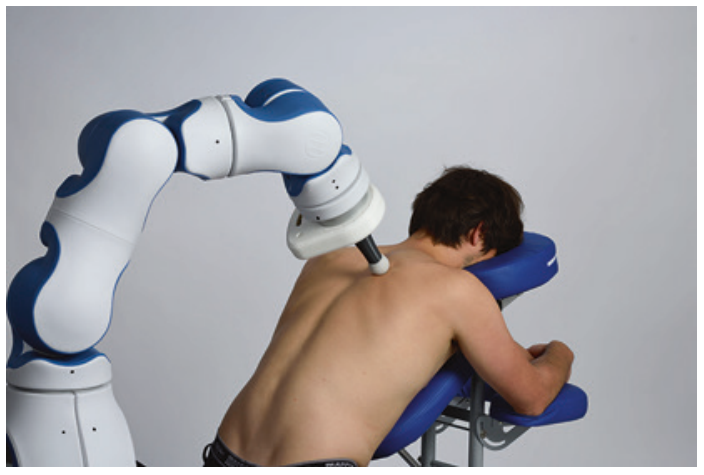

- Kann auf dem Boden, auf Tischen, an der Wand, an der Decke oder auf mobilen Plattformen installiert werden

- Softwarepaket myP

Für die Tagesklinik Glarus wurde der P-Rob, ein Roboterarm, der in unterschiedlichen Varianten und mit verschiedenen Greifern erhältlich ist, mit einer speziellen Applikation, einem weichen Massageball, ausgestattet, um automatisierte Impulsmassagen durchzuführen. P-Rob erkennt mithilfe eines Kamerasystems die vom Personal vorgezeichneten Punkte, welche die gewünschten Behandlungsstellen markieren. Individuell auf die Bedürfnisse und Beschwerden des Patienten abgestimmt, führt P-Rob kreis- oder kegelförmige Bewegungen gezielt aus. Dabei kann die Kraft, mit der die Massage durchgeführt werden soll, von 5 bis $200 \mathrm{~N}$ variiert werden.

So konnte dem bestehenden Personalmangel mit einer automatisierten Lösung entgegengewirkt werden. Der Einsatz von P-Rob in der Tagesklinik ist ein schönes Beispiel für einen kollaborativen Serviceroboter, für den keine Schutzeinrichtung nötig ist und der so den Menschen direkt Hilfestellung leisten kann.

\subsection{Implementierung von Pflegerobotern Lio, P-Care und P-Rob}

Für viele Menschen bringt die Vorstellung, Roboter in Pflege- und Altersheimen zu integrieren, viele Fragezeichen mit sich. Es ist daher wichtig, Schritt für Schritt vorzugehen, um eine einwandfreie Implementation zu ermöglichen. F\&P bietet während der ersten zwei Wochen der Inbetriebnahme Rundumbetreuung und ist auch nach diesem Zeitfenster nach Bedarf zur Stelle, um dem Pflegepersonal bei der Handhabung des Roboters zur Seite zu stehen. Außerdem wird das Verhaltensrepertoire des Roboters gemäß den Anforderungen des Kunden angepasst und Daten von Betreuten, Fachpersonen, den Räumlichkeiten sowie täglichen und wöchentlichen Routineabläufen werden erfasst. Auch Besuchsplanungen können dem Roboter beigebracht werden. 
Um Betrieben die Integration von robotischer Assistenz längerfristig zu erleichtern, bietet F\&P Schulungen für das Pflegepersonal an. Während des ersten Jahres ab der Anschaffung eines Serviceroboters werden auf Wunsch der Institutionen periodisch Anpassungen der Programme vorgenommen sowie Software-Updates offeriert. Zusätzlich werden sowohl die Hardware als auch die Datenbank regelmäßig überprüft.

\subsection{Nutzen der Pflegerobotik}

Die Pflegerobotik bringt einen Nutzen in verschiedensten Bereichen. Zum einen steht bei F\&P Robotics der bewusste Umgang mit Robotern und deren Einsatz als Ergänzung, nicht als Ablösung des Pflegepersonals im Vordergrund. Außerdem steigt die Lebensqualität der Bewohner durch die eingesetzten Serviceroboter merklich, da die betreuten Personen aktiver sind, mehr Anreize zur Kommunikation und zum Trinken erhalten. Schlussendlich bringt die Kollaboration mit Robotern auch wirtschaftliche Vorteile und ermöglicht es Institutionen, sich im kompetitiven Markt einen Platz zu sichern.

Wie Compagna et al. (2009) bemerken, werden sowohl vonseiten der Heimbewohner als auch des Pflegepersonals Vorbehalte gegenüber dem Einsatz von Pflegerobotern geäußert. Dies wurde bei der Studie „Robotik in Betreuung und Gesundheitsversorgung des Schweizer Zentrums für Technologieabklärung“ (2013) bestätigt. Diese Bedenken müssen ernst genommen werden. F\&P Robotics führt deshalb in Alters- und Pflegeheimen Befragungen und Studien durch. Dadurch kann die Interaktion zwischen robotischer Hilfe und menschlichem Pflegepersonal so gestaltet werden, dass die soziale Komponente nicht verloren geht, sondern für Bewohner und Arbeitskräfte gleichermaßen ein Mehrwert entsteht.

\subsubsection{Hilfestellung für Fachpersonen}

Die Roboter von F\&P können das Fachpersonal bei repetitiven und anstrengenden Aufgaben entlasten. Weite Wege, die zurückgelegt werden müssen, oder Transportaufgaben können für Menschen belastend sein. Roboter können diese Aufgaben für das Pflegepersonal übernehmen und so Hilfestellung leisten. Das Personal kann sich dadurch vermehrt auf jene Aufgaben konzentrieren, die direkte menschliche Interaktion sowie hohe pflegerische Kompetenz verlangen.

Statt den Menschen zu ersetzen, ermöglichen Lio und P-Care die gezielte Betreuung älterer Menschen und fungieren als Erweiterung des Pflegepersonals. Durch die Auseinandersetzung mit Technik und deren Einsatz in Wohnheimen mit Hilfe von Robotik werden Pflegeberufe aufgewertet und neue Berufsfelder kreiert. 


\subsubsection{Erhöhen der Lebensqualität der Bewohner}

Die Betreuungsassistenten von F\&P ermöglichen den Bewohnern, autonomer zu leben, wenn sie das möchten. Für viele kleinere Hilfsaufgaben wie etwa das Aufheben von Gegenständen sind die Bewohner nicht länger auf eine Fachperson angewiesen. Die selbstständige Bewältigung solcher Aufgaben kann älteren Menschen helfen, ihre Würde zu bewahren und sich unabhängig zu fühlen. Die Roboter können außerdem rund um die Uhr gerufen werden und den Menschen so auch mitten in der Nacht oder am frühen Morgen Hilfestellung leisten.

Zusätzlich zu praktischen Vorteilen bieten sie den Bewohnern auch Unterhaltung. Der Roboter schlägt Spiele vor, animiert zur Bewegung und ermöglicht Kommunikation. So sorgt er für Spaß und gute Stimmung. Lio und P-Care gewährleisten so Unabhängigkeit und soziale Interaktion und erhöhen die Lebensqualität der betreuten Personen.

\subsubsection{Vorteile für die Organisation von Alters- und Pflegeheimen}

Auch für die Organisation eines Heimes bringt die Integration von Pflegerobotik erhebliche Vorteile. Im kompetitiven Markt für anspruchsvolles Wohnen im Alter ist das Anbieten eines Zusatzservices für die Bewohner ein Alleinstellungsmerkmal. Die Betreuungskosten können mittelfristig durch die Aufteilung der anfallenden Betreuungsaktivitäten in zwei Kategorien reduziert werden. Dabei werden anspruchsvolle Führungs- und Betreuungsfunktionen von qualifizierten Mitarbeiterinnen und Mitarbeitern durchgeführt, welche sich so auf sinnvolle Aufgaben konzentrieren können. Repetitive Routine- und Hilfsfunktionen, die nicht zwingend menschliches Zutun verlangen, werden von intelligenten persönlichen Robotern ausgeführt. So wird die Effizienz gesteigert, ohne die Position des Personals infrage zu stellen. Die Bewohner können jederzeit frei entscheiden, ob und in welchem Maße sie mit Robotern in Kontakt kommen möchten.

Durch die frühzeitige Integration von Betreuungsassistenz-Robotern können Betriebe ihr Wissen im Bereich Robotik aufbauen und Prozesse kennenlernen, welche in Zukunft für eine nachhaltige Leistungserbringung immer wichtiger sein werden.

Roboter sind eine Chance für das Gesundheits- und Sozialwesen. Sie erhöhen die individuelle Autonomie, entlasten das Personal bei repetitiven Tätigkeiten und tragen, beispielsweise durch Sturzerkennung, zur Sicherheit der Bewohner bei. Anbieter im Gesundheitswesen, die sich rechtzeitig mit Robotik beschäftigen, generieren einen Know-how-Vorsprung, der in Zukunft wichtig sein wird.

Dabei müssen sich die Pflegeheime nicht selbst um Technik und Wartung kümmern, sondern überlassen dies den Experten von F\&P Robotics und deren Integrationspartnern. 


\subsection{Anforderungen an die Pflegerobotik}

Um den Nutzen von Pflegerobotern in all den oben erwähnten Bereichen sicherzustellen, müssen verschiedene Anforderungen erfüllt werden. Nur durch eine gute Beziehung zwischen Mensch und Roboter kann die bestmögliche Unterstützung erreicht werden. Aus diesen Gründen setzt F\&P Robotics besonders auf die Erkennung der persönlichen Gewohnheiten und deren laufende Aktualisierung durch die intelligenten Roboter.

\subsubsection{Persönliche Erscheinung und Sicherheit}

Roboter, die von den menschlichen Mitarbeitern akzeptiert werden, generieren einen höheren und nachhaltigeren Nutzen. Die Mensch-Roboter-Kollaboration hält in immer mehr Betrieben Einzug, da die Sicherheit der Produkte stets zunimmt und gleichzeitig der Druck zur Effizienz steigt. F\&P investiert daher in eine persönliche und positive Erscheinung des Roboters. Dabei sind das Design sowie Sicherheitsaspekte, wie zum Beispiel die weiche Außenhülle, entscheidende Faktoren. Auch die einfache Bedienbarkeit des Roboters trägt zu dessen Akzeptanz bei. Es sind keine Programmierkenntnisse notwendig, um den Roboter auf seine Aufgaben vorzubereiten.

\subsubsection{Künstliche Intelligenz}

F\&P hat seine Ursprünge im Artificial Intelligence Lab der Universität Zürich. Die Software, welche zur Bedienung des P-Rob entwickelt wurde, enthält Algorithmen und Konzepte der Künstlichen Intelligenz einschließlich dynamischer Wahrscheinlichkeitsmodelle und Lernverfahren. Dies erlaubt es, Situationen zu erkennen und entsprechend zu agieren. Durch neuronale Netze lassen sich verschiedene Eingangsdaten (z. B. Sensorik) und Erfahrungen kombinieren und Wahrscheinlichkeiten ableiten. Dadurch kann der Roboter selbst entscheiden, welches die wohl beste Lösung in einer spezifischen Situation ist. Die Software unterstützt den Benutzer auch in der einfachen Erstellung von neuen Aufgaben und ermöglicht dem Roboter, Objekte zu lernen und mit diesen entsprechend umzugehen.

\subsubsection{Integriertes und einfach bedienbares System}

Durch Know-how und Erfahrung im Software- und im Hardware-Bereich kann dem Kunden eine komplette Roboterlösung, bestehend aus Roboterarm, Greifer inklusive Sensorik und voll integrierter Steuerung angeboten werden. Somit sinken die Integrationskosten und der Kunde hat für sämtliche Aspekte den gleichen Ansprechpartner. Der P-Rob (als Hauptkomponente der F\&P-Roboter) ist kompakt konzipiert: ein Industriecomputer und ein Netzteil sind direkt in der Basis des Roboters eingebaut. 
Externe Geräte fallen weg, was mobile Anwendungen und das Umstellen des Roboters vereinfacht.

Durch die Kombination der drei Aspekte - Persönlichkeit, künstliche Intelligenz und Integration von Soft- und Hardware - kann F\&P Kunden adressieren, welche die Kostenoder Integrationshürden konventioneller Roboter bisher als zu hoch erachtet haben.

In Zusammenarbeit mit der Psychologin Alina Gasser wurde die Wirkung der F\&P-Pflegeroboter auf ältere Leute 2017 wissenschaftlich untersucht. Dabei konnten verschiedene Aspekte des Roboters, wie zum Beispiel seine optische Wirkung und Sprachfunktionen, in realer Umgebung beobachtet werden. Der folgende Bericht dieser Studie zeigt, dass vor allem Menschen, die stark auf Hilfe angewiesen sind, positiv auf robotische Hilfestellung reagieren.

\subsection{Erfahrungen aus dem Einsatz: Feldstudie im Rahmen einer Masterarbeit}

Erste Feldstudien wurden im Rahmen einer Masterarbeit in Persönlichkeits- und Entwicklungspsychologie der Universität Basel durchgeführt.

\subsubsection{Nutzerzentrierte Forschung Mensch-Roboter-Interaktion}

Wie kürzlich in einer Forschungsarbeit von Bilyea et al. (2017) ausgeführt, haben die meisten technologischen Fortschritte im Bereich der Pflegeroboter in den letzten 12 Jahren stattgefunden. Es ist deshalb wenig überraschend, dass Forschung in diesem Bereich noch in den Kinderschuhen steckt. Frühe Arbeiten von Scopelliti et al. (2005) zeigen, dass die Akzeptanz von und Einstellung zu Robotern, insbesondere bei älteren Menschen, nicht nur von den praktischen Vorteilen abhängen, sondern aus komplexen Beziehungen zwischen den kognitiven, affektiven und emotionalen Komponenten der Menschen und ihren Vorstellungen von Robotern entstehen. Sie fanden heraus, dass ältere Menschen ein höheres Misstrauen gegenüber der Technik hatten und diese auch komplizierter $\mathrm{zu}$ bedienen fanden. In ähnlichen Studien hat sich gezeigt, dass ältere Menschen auch früher aufgaben, wenn sie Schwierigkeiten hatten (Giuliani et al. 2005). Andererseits stellten Stafford et al. (2014) fest, dass Bewohner von Alters- und Pflegeheimen, die physische Einschränkungen hatten, einen Roboter eher benutzten als körperlich gesunde Bewohner. Ähnlich haben Tinker und Lansley (2005) und Pain et al. (2007) dokumentiert, dass ältere Menschen besonders bereit waren, Technologie zu akzeptieren, wenn sie sich an einen spezifischen Bedarf richtete und ihnen somit mehr Unabhängigkeit brachte. Forschung, die als Kernpunkt die Interaktion zwischen Mensch und Roboter hat, war bisher meist beobachtend, unterlag künstlichen Bedingungen und wurde nur mit Fotos und Videos von Robotern durchgeführt, statt experimentell und in realen Umgebungen. Daten wurden überwiegend durch Fragebogen (Giuliani et al. 2005; 
Scopelliti et al. 2005), Nachbearbeitungsinterviews (Birks et al. 2016) oder Fokusgruppen (Broadbent et al. 2012; Wu und Rigaud 2012) gesammelt anstelle von Interaktionen mit Robotern in der Zielumgebung.

Es besteht das Risiko, sich auf Methoden zu verlassen, die nur Daten darüber sammeln, wie die Teilnehmenden die Mensch-Roboter-Beziehung interpretieren. Die tatsächliche Interaktion von Menschen mit einem echten Roboter wird dabei vernachlässigt. Bei der Anwendung im alltäglichen Bereich ist es wichtig, dass sich der Benutzer wohlfühlt, wenn er mit dem Roboter interagiert. Qualitative Bewertungen der Mensch-Roboter-Interaktion sowie Feedback der Teilnehmenden zu ihren Erfahrungen mit dem System sind entscheidend für die Weiterentwicklung zur erfolgreichen Integration von Robotern im Gesundheitswesen. Die Funktionalität ist sicherlich eine der wichtigsten Prädiktoren für die Akzeptanz von Robotern. Es lohnt sich aber, zu prüfen, welche anderen Faktoren die Akzeptanz ebenfalls beeinflussen.

Es gibt nur wenige Studien, zum Beispiel diejenige von Fischinger et al. (2013), welche die Interaktion zwischen Menschen und Robotern analysieren. Die Aufgaben des von ihnen entwickelten Roboters HOBBIT waren die Reinigung des Bodens von herumliegenden Objekten, Objekte erlernen, ein Objekt bringen, das Erkennen einer am Boden liegenden Person, aber auch Unterhaltung mit Musik, Videos und Spielen.

Aus der nonverbalen Kommunikation kann viel zu einer Person und deren Beziehung zu ihrem Kommunikationspartner herausgelesen werden (Argyle 2013). Zum Beispiel neigen Menschen dazu, aktive Körperbewegungen als Zeichen von Engagement und Sympathie für andere zu interpretieren (Maxwell et al. 1985). Es liegt daher nahe, dass körperliche Bewegungen sich ebenfalls auf die Art und Weise auswirken könnten, wie ein Roboter wahrgenommen wird. Allerdings haben sich bisher nur wenige Studien mit dieser Frage beschäftigt. Bis anhin hat die Forschung herausgefunden, dass die Teilnehmenden dazu neigten, einen Roboter als lebensechter oder menschenähnlicher zu empfinden, wenn er den Kopf neigt (Mara und Appel 2015), die Blicke anderer teilt (Scassellati 2000) oder Lächeln und Nicken imitiert (Takano et al. 2008). Keine dieser Studien wurde mit älteren Teilnehmenden durchgeführt.

\subsubsection{Forschungsfragen}

Folgenden Forschungsfragen wurde in der Masterarbeit nachgegangen:

- Wie wirken sich persönliche Faktoren und bereits bestehende Denkansätze auf die Reaktion auf einen Roboter aus?

- Wie behandeln ältere Menschen einen Roboter und was sind ihre Herausforderungen bei der Interaktion?

- Wie verändern körperliche Bewegungen eines Roboters die Art und Weise, wie ältere Menschen mit dem Roboter interagieren? 


\subsubsection{Testdesign}

Um die Forschungsfragen zu untersuchen, wurde eine Studie mit „Between-SubjectsDesign“ durchgeführt. Dies bedeutet, dass jede Person nur einer Versuchsbedingung zugeteilt wurde, nämlich entweder mit oder ohne Körperbewegung des Roboters. Die abhängige Variable war die Bewertung auf der Godspeed-Anthropomorphismus-Skala (Bartneck et al. 2009). Mehrere Variablen wurden quantitativ bewertet. Diese waren Interaktionsdauer, Anzahl der Wörter, die die Teilnehmenden insgesamt zu dem Roboter gesprochen haben, Anzahl der höflichen Worte wie „Bitte“ oder „Danke“. Teilnehmerbezogene Variablen wie das Geschlecht, Alter, und ob eine Mobilitätsbeeinträchtigung die zusätzliche Unterstützung erforderte (,,keine Mobilitätsbeeinträchtigung“, „Gehhilfe“, „Rollstuhl“), wurden aufgenommen. Die Interaktion wurde transkribiert und qualitativ analysiert. Die Studie wurde in zwei verschiedenen Alters- und Pflegeheimen, in der „,natürlichen Umgebung“ der älteren Personen, durchgeführt. Die Studie fand teilweise in den privaten Räumlichkeiten der Teilnehmenden statt wie auch in einem der Gemeinschaftsräume des Pflegeheims.

\subsubsection{Teilnehmende}

Für die Rekrutierung wurden drei Pflegeheime in der Schweiz kontaktiert, von denen zwei zusagten. Die Leitenden der Einrichtungen erhielten einen groben Plan des Testablaufs und grundlegende Informationen über den Roboter. Diese Informationen wurden dann an die Bewohner und Bewohnerinnen weitergeleitet. Die Leitenden wählten nach eigenem Gutdünken aus, wen sie für die Teilnahme an der Studie anfragten. Ältere Personen mit Demenz wurden aufgrund ethischer Überlegungen ausgeschlossen. Das Interesse war so groß, dass statt den geplanten 12 Testdurchläufen insgesamt 18 eingeplant wurden. Schlussendlich nahmen 12 Bewohnerinnen und 5 Bewohner im Alter von 70 bis 96 (durchschnittlich 85) Jahren an der Studie teil. In 15 Fällen hatten sie altersbedingte Mobilitätsbeeinträchtigungen (sieben mit Rollator, acht mit Rollstuhl). Zwei Teilnehmende waren körperlich völlig unabhängig.

\subsubsection{Der Roboter}

Der Designprozess wurde, wie von Vincze et al. (2016) vorgeschlagen, angepasst, um einem benutzerzentrierten Ansatz zu folgen, der die Bedürfnisse und Erwartungen der potenziellen Benutzergruppe berücksichtigt. Für die Versuche in dieser Studie wurde in enger Zusammenarbeit zwischen Ingenieuren und Psychologen ein Skript, bestehend aus Sprach- und Körperbewegungen, vordefiniert. Das Skript wurde als sogenanntes Wizardof-Oz-Studiendesign ausgeführt (Dahlbäck et al. 1993). Der Roboter wurde aus der Ferne gesteuert, um die korrekte Positionierung des Roboters vor den Teilnehmenden sicherzustellen. Der Roboter wurde von einer Technikerin gelenkt, die sich hinter dem Teilnehmenden positionierte. Die Vorteile dieser Form sind ein hohes Maß an Kontrolle über die Manipulationen der Studie, aber auch den Roboter schneller reagieren zu lassen, als er es normalerweise könnte, um auch in Bezug auf die Reaktionszeit menschenähnlicher 
zu sein. Des Weiteren ermöglicht es dieses Design, durch den ferngesteuerten Roboter für alle Teilnehmenden eine vergleichbare Situation zu schaffen.

\subsection{Eingabemodalität}

Fischinger et al. (2016) ließen ihre Teilnehmenden frei wählen, welche Eingabemodalität sie bei der Interaktion mit einem Roboter verwenden wollten. Obwohl die meisten Teilnehmenden zu Beginn Sprache als Eingabemodalität für die Interaktion mit dem Roboter wählten, wechselten sie oft auf den Touchscreen, um dem Roboter ein Objekt beizubringen. Dies hängt damit zusammen, dass diese Aufgabe für die Teilnehmenden am schwierigsten war. Nach sechs kooperativen Aufgaben bewerteten die Teilnehmenden die Arbeitsweise, die sie bevorzugten. Die Ergebnisse zeigten folgende Reihenfolge: Sprachbefehle (49\%), Touchscreen $(42,9 \%)$ und Gesten $(6,1 \%)$.

Demzufolge wurde für die aktuelle Studie Sprache als Eingabemodalität während der Interaktion mit dem Roboter gewählt. Um die Interaktion möglichst einfach zu halten, wurde nur eine Modalität verwendet. Die Ausgabemodalität wurde deshalb auch auf Sprache konfiguriert.

\subsection{Roboterstimme: Geschlecht und Dialekt}

Studien von Nass et al. (1997) und Eyssel et al. (2012) ergaben, dass ein Roboter positiver wahrgenommen wird, wenn der Eindruck eines gleichgeschlechtlichen Gegenübers entsteht. Weil der Prozentsatz von Frauen in der Zielgruppe der älteren Personen höher ist, wurde für den Roboter eine weibliche Stimme gewählt.

Die Stimme, die für das Skript benutzt wurde, wurde aufgezeichnet. Es war die natürliche Sprache einer jungen Frau ohne professionelle Ausbildung in diesem Bereich, jedoch im Dialekt der Region gesprochen. Der Name der Sprecherin war Angela. Dieser Name wurde auch für den Roboter benutzt.

\subsection{Körperliche Bewegungen}

Körperbewegungen wurden als Bewegungen definiert, die nicht erforderlich waren, um eine Aufgabe abzuschließen. Das Bücken des Roboters und das Bewegen der Greifer um ein Objekt herum wurden beispielsweise benötigt, um ein Objekt aufzunehmen, und daher nicht als körperliche Bewegung bezeichnet. Die Körperbewegungen wurden verwendet, um den Roboter lebensechter erscheinen zu lassen: Verbeugen beim Grüßen oder Abschiednehmen, Bewegen des Oberkörpers, um einen Gegenstand aus verschiedenen Richtungen anzuschauen, Bewegungen zum Takt von Musik und ein Abdrehen des obersten Gelenks, nachdem eine Frage gestellt wurde, um Interesse zu signalisieren (imitiert eine Kopfneigung). Die Hälfte der Teilnehmenden interagierte mit dem Roboter mit diesen Körperbewegungen, während die andere Hälfte mit dem Roboter ohne diese körperlichen Bewegungen interagierte. Angelehnt an die Studien von Vincze et al. (2016), Robinson et al. (2014), Tinetti und Williams (1997), Cumming et al. (2000) und Fischinger et al. (2016) bestand die Interaktion aus Aufgaben, die mit dem Roboter gelöst wurden. Die Teilnehmenden brachten dem Roboter ein Objekt bei, 
wählten, ob sie als Gegenleistung von dem Roboter ein Lied hören wollten, legten das neue Objekt auf den Boden und befahlen dem Roboter, das Objekt aufzuheben.

\subsubsection{Messinstrumente}

Die verwendeten Messinstrumente waren halb strukturierte Interviews, Fragebogen und Beobachtungsprotokolle sowie Sprach- und Videoaufnahmen der Versuche. Die Aufnahmen wurden transkribiert und es wurde eine thematische Analyse nach Terry et al. (2017) durchgeführt. Um zu beurteilen, wie stark der Roboter ohne menschliche Form, aber mit menschenähnlichen Eigenschaften wahrgenommen wurde, wurde die Godspeed-Anthropomorphismus-Skala (Bartneck et al. 2009) verwendet. Anthropomorphismus kann auf verschiedene Arten konzeptualisiert und gemessen werden - von einer einfachen Bewertung von Menschenähnlichkeit auf einer eindimensionalen Skala bis hin zu breiteren und weniger konkreten Konzeptualisierungen einschließlich Geist, Emotionalität, Absicht, Bewusstsein und freiem Willen (Waytz et al. 2010). Aufgrund der Tatsache, dass die meisten Teilnehmenden über 80 Jahre alt sind und Fragebogen in der Regel am Ende einer Studie platziert werden, wurde mit Rücksicht auf Ermüdung und Konzentrationsfähigkeit die Godspeed-Anthropomorphismus-Skala (Bartneck et al. 2009) mit nur fünf Items verwendet (Cronbachs Alpha=,76). Die Skala verwendet das Abwägen zwischen folgenden Wortpaaren: gefälscht oder natürlich, maschinenähnlich oder menschenähnlich, unbewusst oder bewusst, künstlich oder lebensecht, sich starr bewegend oder sich elegant bewegend. Darüber hinaus wurde kompetent oder inkompetent und auch schnell oder langsam als semantisches Differenzial auf einer 5-Punkte-Skala bewertet.

\subsection{Studienablauf}

\subsubsection{Erster Test}

Ein erster Test wurde mit drei älteren Personen durchgeführt, vor allem, um technische Details zu prüfen. Die Haupterkenntnisse waren, dass die Stimme des Roboters so laut und klar wie möglich sein sollte, sodass auch ältere Menschen mit Hörschwierigkeiten eine bessere Chance hatten, zu verstehen, was der Roboter sagte, und dass die gewählte Spracherkennung nicht gut genug war, um den lokalen Dialekt zu verstehen.

Es war sehr überraschend zu sehen, wie kommunikativ sich die älteren Personen gegenüber dem Roboter verhielten. Dieses Verhalten machte das Abschätzen von möglichen Antworten sehr schwierig. Der Wortlaut des Skripts wurde leicht angepasst, um klarere Anweisungen zu geben. Das Skript wurde ebenfalls angepasst, um den Benutzern mehr Raum zum Reden zu geben. Es wurde folgender Satz eingebaut: „Erzählen Sie mir doch etwas über sich“ (Schweizerdeutsch: „Verzelled Sie mir doch öpis über sich“). Der Satz wurde insgesamt zwei Mal in den Dialog eingebaut, einmal am Anfang der Konversation und einmal am Schluss. Die Hypothese war, dass die ältere Person dem Roboter beim 
zweiten Mal mehr persönliche Dinge preisgeben würde als beim ersten Kontakt, sofern sie den Roboter aufgrund der Interaktion mochte.

\subsubsection{Zweiter Test}

Im ersten Teil wurden die Teilnehmenden in ihren privaten Räumen besucht, wo der erste Teil des Interviews stattfand. Sie wurden dann zum Gemeinschaftsraum der Bewohner begleitet, wo sie nach einer kurzen Einführung mit dem Roboter in der Interaktion ein paar vorgegebene Aufgaben lösten. Die Interviewerin, ein Assistent und die Technikerin waren immer anwesend. In einigen Fällen war auch Pflegepersonal anwesend, das aus dem hinteren Teil des Saals bei der Interaktion zuschaute. Die Technikerin saß hinter dem Teilnehmenden und steuerte das Herumfahren des Roboters.

Nachdem sichergestellt war, dass sich die Teilnehmenden wohlfühlten, wurde das Skript gestartet. Die eine Hälfte der Teilnehmenden erlebte den Roboter mit den Körperbewegungen, die andere Hälfte ohne. Die Teilnehmenden wurden den Bedingungen zufällig zugeteilt. Der erste Schritt des Roboters war es, sich dem Teilnehmenden zu nähern, ihn zu begrüßen und sich selbst als Angela vorzustellen. Die Aufgaben während der Interaktion waren, dem Roboter ein neues Objekt beizubringen, zu wählen, ob als Gegenleistung für diese Hilfe ein Lied gehört werden wollte, das neu gelernte Objekt auf den Boden zu legen und dem Roboter zu befehlen, den Gegenstand aufzuheben. Nach diesen kooperativen Aufgaben verabschiedete sich der Roboter und fuhr weg. Die Teilnehmenden berichteten dann im nächsten Teil über ihre Erfahrungen und bewerteten den Roboter auf der Godspeed-Anthropomorphism-Scale mit dem Interviewer. Am Ende erhielten die Teilnehmenden als Dankeschön ein kleines Konfekt und wurden gefragt, ob sie gerne ein Foto von sich selbst mit dem Roboter zusammen möchten, um es als Souvenir zu behalten.

\subsection{Feedback von Benutzern und deren Implikationen}

Ziel der Studie war es, das Zusammenwirken einer älteren Person mit einem Roboter $\mathrm{zu}$ beobachten, zu erforschen und zu beschreiben, und zwar anhand von Interaktionen mit dem Roboter P-Rob in einem Alters- und Pflegeheim in einer regionalen Schweizer Stadt. Dieser Abschnitt fasst die Auswirkungen der Ergebnisse und Antworten auf die Forschungsfragen zusammen.

\subsubsection{Konsequenzen von persönlichen Faktoren und bereits bestehenden Mindsets}

In erster Linie waren die älteren Personen sehr neugierig. Dass eine größere Anzahl von älteren Menschen teilnehmen wollte, als ursprünglich geplant war, zeigt, wie interessiert sie waren. Persönliche Faktoren wie körperliche Beeinträchtigung hatten einen 
Einfluss darauf, als wie nützlich die älteren Personen den Roboter wahrnahmen. Zwei Teilnehmende sahen den Roboter als Personifikation ihrer Unfähigkeit: „,Danke viel Mal. Ja. Auf Wiedersehen. Hoffentlich nicht sehr bald.“ („Danke vielmal. Jo. Uf Wiederseh. Hoffed mir, nöd so gschwind wieder." $)^{1}$ Dies steht im Einklang mit den Befunden von Fischinger et al. (2016), nach denen einige Teilnehmende der Meinung waren, dass der Roboter in der Tat hilfreich sein könnte, aber dass sie selbst noch zu gesund oder aktiv waren, als dass sie momentan ein solches Gerät bräuchten. Möglicherweise war dies der Grund, weshalb zwei Teilnehmende am Schluss der Studie kein Foto mit dem Roboter mitnehmen wollten. Das wäre vergleichbar damit, ein Foto von jemandem im Rollstuhl zu machen, obwohl derjenige eigentlich keinen braucht. Die körperliche Unabhängigkeit ist ihnen wichtig und dies möchten sie auch zeigen.

Es ist ermutigend, dass diejenigen Teilnehmenden, die vermutlich den größten Bedarf an Unterstützung eines Roboters haben, den Roboter am meisten akzeptieren. Jedoch könnte die Stigmatisierung, die mit der Assoziierung des Roboters mit einem Verlust der Unabhängigkeit einhergeht, die zukünftige Einstellung von Personen mit weniger schweren Beeinträchtigungen negativ beeinflussen. Dies, obwohl letztere von der Unterstützung profitieren könnten, um beispielsweise Verletzungen vorzubeugen.

Es sollte daher versucht werden, hervorzuheben, wie ein Roboter auch für ältere Menschen ohne oder mit nur geringer Beeinträchtigung nützlich sein kann. Viele Roboter enthalten bereits Unterhaltungssysteme oder Telekommunikationstechnologien. Ältere Personen mit der Einstellung, dass Roboter ,Zerstörer des menschlichen Kontakts“" seien, nahmen aufgrund von Neugierde trotzdem freiwillig an der Studie teil. Dies kann bedeuten, dass ältere Menschen, die generell gegen den Einsatz von Robotern in Gesundheitseinrichtungen sind, nach einer begleiteten Einführung und Anleitung zum Umgang mit dem Roboter eine positivere Sichtweise auf die Robotik adaptieren würden. Vielleicht kann der Roboter auch als Spielzeug betrachtet werden und als etwas, das Spaß bereitet, ohne ihn zu brauchen. Dies könnte helfen, den Roboter als nützlich zu sehen, auch wenn man keine altersbedingten Einschränkungen hat. Es wäre eine weitere Studie wert, ob es vorteilhaft wäre, wenn der Roboter mehr als Quelle der Unterhaltung empfunden wird, statt nur als ein Gerät, welches Selbstständigkeit im Alltag unterstützt.

\subsubsection{Umgang mit dem Roboter und Herausforderungen bei der Interaktion}

\subsubsection{Folgen mangelnder Erfahrung}

Keiner der Teilnehmenden hatte Erfahrung im Umgang mit Robotern. Dies beeinflusste das Verhalten der älteren Personen vor allem in drei Situationen: 1) wenn der Roboter zwei verschiedene Möglichkeiten bot, 2) wenn die ältere Person einen spezifischen

\footnotetext{
${ }^{1}$ Direktes Zitat von einem Teilnehmer, das in seiner deutschen Übersetzung sowie wie dem ursprünglichen schweizerdeutschen Dialekt notiert wurde.
} 
Befehl geben sollte, und 3) in ihrer Einschätzung, wie kompetent der Roboter war. Ältere Menschen, die keine Erfahrung in der Kommunikation mit Robotern haben, sind es nicht gewohnt, präzise und kurze Befehle zu geben. Ihnen fehlt das Wissen, dass eine Maschine hauptsächlich darauf ausgelegt ist, auf Hinweise zu reagieren, die aus vordefinierten Wörtern oder Sätzen bestehen. Folglich waren einige der älteren Menschen in dieser Studie sehr gesprächig und plauderten überraschend offen mit dem Roboter, als ob es ein menschliches Wesen wäre, obwohl sein Design das Gegenteil andeutete.

\section{Auswirkungen}

Ein Roboter muss in der Lage sein, auf sehr viele verschiedene Eingaben zu reagieren. Für die Spracherkennung bedeutet dies, nicht nur auf einzelne Wortzuweisungen zu reagieren, sondern auf den Inhalt ganzer Sätze. Idealerweise kann der Roboter entscheiden, ob der Inhalt positiv oder negativ ist, und ihn mit dem Gesichtsausdruck des Benutzers vergleichen, um die korrekte Erkennungsrate zu erhöhen.

Gerade dieser Mangel an Erfahrungswissen auf der Seite der älteren Personen in dieser Studie ermöglichte es ihnen, viel offener für das zu sein, was der Roboter leisten konnte. Da die älteren Personen keine vorherige Erfahrung hatten, wussten sie nichts über den Umfang der heutigen technischen Möglichkeiten. Dies spiegelte sich auch in der Tatsache wider, dass die Teilnehmenden den Roboter als kompetent empfanden, selbst wenn es zwei oder in einem Fall sogar drei Versuche dauerte, ein Objekt aufzuheben. Sie fanden den Roboter auch nicht langsam, selbst wenn der Roboter in einem Fall fast drei Minuten benötigte, um den Gegenstand aufzuheben. Es ist wahrscheinlich, dass sie, wenn sie mehr Erfahrung hätten, erwartet hätten, dass der Roboter schneller ist oder Funktionalitäten zur Spracherkennung hat. Weitere Untersuchungen sind erforderlich, um herauszufinden, wie das Design eines Roboters die Wahrnehmung und die Erwartungen eines Benutzers beeinflusst.

\subsubsection{Höflichkeit}

Die älteren Menschen in dieser Studie waren insgesamt sehr höflich zu dem Roboter und sagten „Danke“ und „Bitte“. Einige ältere Menschen benutzten das Pronomen „Sie“, wenn sie den Roboter ansprachen. Dies unterstützt experimentelle Befunde von Nass (2004), die zeigten, dass Menschen menschliche Qualitäten in Computern wahrnehmen, die sie eigentlich nicht haben. In dieser Studie waren sie nicht nur höflich, sondern auch interessiert am Musikstück, welches der Roboter als sein Lieblingslied deklarierte. Dies impliziert, dass sie akzeptieren, dass der Roboter eine Persönlichkeit mit eigenen Präferenzen haben kann.

Im Vergleich unserer Ergebnisse mit ähnlichen Literaturarbeiten haben wir festgestellt, dass auch in unserer Studie ältere Menschen den Robotern menschliche Züge wie Höflichkeit und Humor zuschrieben und erwarteten, dass sie sich so intelligent wie Menschen verhalten, obwohl sie wussten, dass der Roboter eine Maschine ist. Das Verhalten von Robotern, wie beispielsweise die Verwendung von Körperbewegungen, beeinflusst höchstwahrscheinlich, wie lebensecht der Roboter wahrgenommen wird. In unserer 
Studie genossen die Teilnehmenden die Interaktion mit dem Roboter und waren beeindruckt von seiner Technologie, was die Ergebnisse anderer Home-Studien bestätigt, wie zum Beispiel diejenige von Fischinger et al. (2013).

\subsection{Auswirkungen}

Ein Roboter sollte persönliche Eigenschaften oder Präferenzen wie zum Beispiel ein Lieblingslied haben, um die Interpretation menschlicher Qualitäten zu erleichtern.

\subsubsection{Herausforderungen aufgrund von Hörschwierigkeiten}

Damit ältere, gehörschwache Menschen mit dem Roboter interagieren konnten, musste die Sprachausgabe so laut und deutlich wie möglich sein. Allerdings war nicht immer klar, was der Roboter wollte, selbst wenn sie akustisch verstanden, was dieser sagte. Die Teilnehmenden wandten sich dann jeweils an den Interviewer. Dies war beispielsweise manchmal der Fall, als der Roboter ihnen sagte, sie sollen ein Objekt vor das „Gesicht“ des Roboters halten.

\subsection{Auswirkungen}

Das Kreieren eines Roboters für eine spezifische Bevölkerungsgruppe, wie ältere Menschen, bringt besondere Herausforderungen mit sich. Es ist essenziell sicherzustellen, dass das Design mögliche altersbedingte Einschränkungen, wie z. B. Hörverlust, berücksichtigt, indem sichergestellt wird, dass der Roboter laut und deutlich genug spricht. Dass jedem Roboter alle wichtigen Objekte gelehrt werden müssen, könnte vermieden werden, indem die Daten eines Objekts, beispielsweise einer Brille, gesammelt, gespeichert und einem anderen Roboter mitgegeben werden. Somit könnte der nächste Roboter, der eingesetzt wird, bereits mit einer größeren „Objektliste“, wie Frennert et al. (2017) es nennen, beginnen. Wichtige Aufgaben wie das Holen und Aufheben von Objekten sind an die Besonderheiten eines Objekts wie Größe und Form gebunden, die der Roboter entweder bereits in seiner Datenbank gespeichert hat oder neu dazulernen kann. Um die Leistung dieser Hilfestellungen zu verbessern, muss der Roboter ein Objekt und den Namen, bei dem der Benutzer es nennt, kennen. Da die älteren Menschen in dieser Studie jedoch sehr offen mit dem Roboter waren, ist es vorstellbar, dass sie dem Roboter sehr persönliche Gegenstände beibringen würden, die dann automatisch in ihrer Datenbank gespeichert würden. Dieses Thema könnte ethische Fragen in Bezug auf die Sammlung von Daten über persönliche Gegenstände oder sogar Gegenstände, die gesetzlich verboten sind, aufwerfen. Dies bringt ethische Implikationen mit sich, die im Designprozess sorgfältig berücksichtigt werden sollten.

\subsubsection{Konsequenzen körperlicher Bewegungen und anderer Robotermerkmale für Anthropomorphismus und Akzeptanz}

Ähnlich wie in den Ergebnissen anderer Studien (Broadbent et al. 2009; Syrdal et al. 2008; Walters et al. 2008) erwarteten die Teilnehmenden, dass der Roboter eine bestimmte Persönlichkeit hat. Sie wollten mit der Information, welches das Lieblingslied 
des Roboters war, überrascht werden. Sie wollten dem Roboter beim Lernen und seiner Entwicklung helfen. Der Roboter sollte spontan, intelligent und sozial sein. Qualitative Beobachtungen ergaben, dass die Teilnehmenden die Gesten des Roboters nachahmten. Teilnehmende verbeugten sich, wenn der Roboter sich verbeugte, und wenn der Roboter zu seiner Lieblingsmusik tanzte, ahmten die Teilnehmenden manchmal seine seitlichen Bewegungen im Stuhl nach oder wippten mit ihrem Fuß im Takt. Während die geringe Stichprobengröße keine quantitativen Interpretationen der Daten zulässt, sind diese Ergebnisse sicherlich eine weitere Untersuchung mit einer größeren Stichprobe wert.

\subsection{Auswirkungen}

Die Ergebnisse der Forschung in der Mensch-Mensch-Interaktion von Ramseyer und Tschacher (2011) legen nahe, dass nonverbale Synchronität die von den Patienten selbst berichtete Qualität der Beziehung verkörpert. Solche Bewegungen können dem Roboter, auch wenn sie für seine Funktionalität, Aufgaben auszuführen, nicht notwendig sind, einen einzigartigen Charakter verleihen und ihn dadurch menschlicher erscheinen lassen und die soziale Interaktion fördern.

\subsubsection{Der lokale Dialekt}

Eine weitere Frage für zukünftige Forschung ist diejenige nach dem Einfluss lokaler Dialekte auf Anthropomorphismus und die Akzeptanz von Robotern. Die Teilnehmenden dieser Studie waren im Allgemeinen sehr freundlich und offen gegenüber dem Roboter.

\subsection{Auswirkungen}

Die Untersuchung von Auswirkungen von Dialekten auf Mensch-Roboter-Interaktionen beschränkt sich bisher hauptsächlich auf die englischsprachige Welt. Die Präferenz für Ähnlichkeit ist aber auch auf die Wahrnehmung von Maschinen anwendbar. Als Teilnehmende einen Roboter betrachteten, der mit einer Stimme ihres eigenen Geschlechts sprach, wurde er als menschlicher beurteilt (Eyssel et al. 2012; Nass et al. 1997). Zudem wurden gegenüber einem Roboter, der im eigenen Dialekt spricht, mehr positive Gefühle geäußert als bei anderen Dialekten (Tamagawa et al. 2011).

Angesichts der Bedeutung von Dialekten für die Identität, vor allem in Bereichen mit sehr spezifischen Dialekten, ist es möglich, dass das Gespräch mit dem Roboter im lokalen Dialekt die Bereitschaft der Teilnehmenden erhöht haben könnte, mit dem Roboter zu interagieren und ihn in einem positiven Licht zu sehen. Eine nähere experimentelle Untersuchung dieser Frage wäre sehr interessant für Roboter, die weltweit eingesetzt werden sollen.

\subsubsection{Weitere wichtige Erkenntnisse: Die Notwendigkeit der Anpassungsfähigkeit}

Die Teilnehmenden dieser Studie wiesen eine große Varianz an körperlichen Fähigkeiten auf. Einige waren noch relativ unabhängig, während andere selbst bei den kleinsten körperlichen Aufgaben Hilfe brauchten. 


\subsection{Auswirkungen}

In Übereinstimmung mit Forlizzi et al. (2004) legen die Ergebnisse dieser Studie nahe, dass ein Gesundheitsroboter sehr anpassungsfähig sein muss, um älteren Menschen mit verschiedenen Beeinträchtigungen in verschiedenen Stadien Hilfestellung leisten zu können. Ein unabhängiger 90-Jähriger sollte sich nicht darauf verlassen, dass der Roboter Gegenstände für ihn holt. Die Lebensqualität eines älteren Menschen mit körperlichen Beeinträchtigungen würde jedoch stark zunehmen, wenn der Roboter beispielsweise Fingerfood anbieten könnte. Wir altern nicht nur alle physisch anders, sondern jedes Individuum altert in Abhängigkeit von mentaler und körperlicher Stärke. Dies kann sich außerdem von Tag zu Tag stark unterscheiden. Wie Frennert et al. (2017) anmerken, gibt es verschiedene Aspekte, die bei der Definition der Aufgaben des Roboters für ein Individuum berücksichtigt werden müssen: nicht nur, was für den Einzelnen am bequemsten ist, sondern was die Lebensqualität und den Lebensstandard verbessert. Auf der einen Seite sollte ein Roboter bei den täglichen Aktivitäten helfen und auf der anderen Seite sollte er älteren Menschen nicht die Möglichkeit nehmen, sich zu bewegen. Der Roboter sollte die Autonomie eines Individuums auf lange Sicht nicht einschränken. Dies würde bedeuten, dass ein Roboter eine Person genau überwachen sollte, um beispielsweise entscheiden zu können, wie viel Übung bereits ausgeführt wurde, und davon zu unterscheiden, welche Art von Verhalten für den Roboter geeignet ist. Wenn die ältere Person zum Beispiel kürzlich ein Bewegungsprogramm abgeschlossen hat, würde der Roboter der Anfrage, ein Buch zu holen, nachgehen, anstatt den Benutzer zu ermutigen, den Roboter zu begleiten, während er das Objekt holt. Der Roboter könnte auch die Menge der eingenommenen Flüssigkeit überwachen. Besonders ältere Menschen neigen dazu, zu wenig zu trinken. Der Roboter kann von Zeit zu Zeit ein Glas Wasser anbieten. Viele Daten müssten mit der Hilfe von medizinischem Personal gesammelt werden, um die Aufnahme von Wasser und Nahrung, oder wie oft sie sich bewegen, genau zu überprüfen. Es muss noch detaillierter erforscht werden, wie Roboter den Menschen genau überwachen können und sollen.

\subsection{Ausblick}

\subsubsection{Gesellschaftsentwicklung}

Aktuelle gesellschaftliche Entwicklungen zeigen, dass die Pflegerobotik in Zukunft einen immer wichtigeren Stellenwert haben wird. In Deutschland waren 2015 insgesamt $9 \%$ mehr Menschen pflegebedürftig als 2013 (Statistisches Bundesamt 2017). Die zunehmend höhere Lebenserwartung sowie ein wachsendes Gesundheitsbewusstsein der Bevölkerung stellen neue Anforderungen an die Technologie. Außerdem droht gleichzeitig ein Mangel an Fachpersonal im Pflegebereich. Um diesem Mangel entgegenzuwirken, empfehlen Klein et al. (2018), ,erfolgreich getestete Modellassistenzlösungen flächendeckend zu implementieren“. Dazu gehören auch verbesserte IT-Infrastrukturen 
in den entsprechenden Gebäuden. Eine frühzeitige Auseinandersetzung mit den Möglichkeiten der Robotik ist für Pflege- und Altersheime essenziell, um auch zukünftig die bestmögliche Betreuung für ihre Bewohnerinnen und Bewohner zu garantieren.

\subsubsection{Bedarf an Pflegerobotik}

Die Gesellschaftsentwicklung und der damit verbundene Bedarf an Pflegerobotik hat direkten Einfluss auf die Funktionen von Pflegerobotern. F\&P Robotics ist überzeugt, dass sich nur diejenigen technischen Systeme durchsetzen werden, welche einen konkreten Nutzen im Alltag stiften, sowohl für die betreuten Personen als auch die Fachpersonen. Dazu kann die Erhöhung der Autonomie gehören, das Schaffen von mehr Freiraum für menschliche Interaktionen, aber auch die Prävention und Erkennung von Gefahrensituationen.

In Deutschland, in Österreich und in der Schweiz baut F\&P gemeinsam mit Partnern proaktiv Pionieranwendungen auf, die den Nutzen des Einsatzes von Robotik zukunftsorientiert und nutzerzentriert demonstrieren. Dabei können sich die betreuenden Personen voll auf die zwischenmenschlichen und anspruchsvollen Aufgaben konzentrieren, während der Pflegeroboter gezielt rund um die Uhr unterstützt. Die bereits umgesetzten Anwendungen orientieren sich dabei stets an einem oder mehreren konkreten Bedürfnissen der Praxis.

\section{Literatur}

Adami, I., Antona, M., \& Stephanidis, C. (2016). Home trials of robotic systems: Challenges and considerations for evaluation teams. In International conference on universal access in human-computer interaction (S. 291-301).

AhYun, K. (2002). Similarity and attraction. Interpersonal communication research: Advances through meta-analysis, 145-168.

Andrade, A. O., Pereira, A. A., Walter, S., Almeida, R., Loureiro, R., Compagna, D., et al. (2014). Bridging the gap between robotic technology and health care. Biomedical Signal Processing and Control, 10, 65-78.

Argyle, M. (2013). Bodily communication. Routledge.

Bartneck, C., Kulić, D., Croft, E., \& Zoghbi, S. (2009). Measurement instruments for the anthropomorphism, animacy, likeability, perceived intelligence, and perceived safety of robots. International Journal of Social Robotics, 1(1), 71-81.

Bilyea, A., Seth, N., Nesathurai, S., \& Abdullah, H. (2017). Robotic assistants in personal care: A scoping review. Medical Engineering \& Physics, 49,1-6.

Birks, M., Bodak, M., Barlas, J., Harwood, J., \& Pether, M. (2016). Robotic seals as therapeutic tools in an aged care facility: A qualitative study. Journal of aging research, 2016.

Bloom, D. E., Chatterji, S., Kowal, P., Lloyd-Sherlock, P., McKee, M., Rechel, B., . . S Smith, J. P. (2015). Macroeconomic implications of population ageing and selected policy responses. The Lancet, 385(9968), 649-657. 
Bohren, J., Rusu, R. B., Jones, E. G., Marder-Eppstein, E., Pantofaru, C., Wise, M., ... Holzer, S. (2011). Towards autonomous robotic butlers: Lessons learned with the PR2. In 2011 IEEE International conference on robotics and automation (ICRA) (S. 5568-5575).

Braun, V., \& Clarke, V. (2006). Using thematic analysis in psychology. Qualitative research in psychology, 3(2), 77-101.

Broadbent, E. (2017). Interactions with robots: The truths we reveal about ourselves. Annual Review of Psychology, 68, 627-652.

Broadbent, E., Kerse, N., Peri, K., Robinson, H., Jayawardena, C., Kuo, T., et al. (2016). Benefits and problems of health-care robots in aged care settings: A comparison trial. Australasian Journal on Ageing, 35(1), 23-29.

Broadbent, E., Stafford, R., \& MacDonald, B. (2009). Acceptance of healthcare robots for the older population: Review and future directions. International Journal of Social Robotics, 1(4), 319-330.

Broadbent, E., Tamagawa, R., Patience, A., Knock, B., Kerse, N., Day, K., et al. (2012). Attitudes towards health-care robots in a retirement village. Australasian Journal on Ageing, 31(2), $115-120$.

Bundesamt für Statistik (Hrsg.). (2017). Pflege im Rahmen der Pflegeversicherung Deutschlandergebnisse (Statistisches Bundesamt/Januar 2017). Pflegestatistik 2015. Wiesbaden: Bundesamt für Statistik.

Chang, S., \& Sung, H. (2013). The effectiveness of paro robot therapy on mood of older adults: A systematic review. International Journal of Evidence-Based Healthcare, 11(3), 216.

Compagna, D., Derpmann, S., Mauz, K., \& Shire, K. A. (2009). Zwischenergebnisse der Bedarfsanalyse für den Einsatz von Servicerobotik in einer Plegeeinrichtung: Routine- vs. Pflegetätigkeiten. In: Working Brief 7/2009. https://www.uni-due.de/imperia/md/content/wimi-care/ wb_7_.pdf. Zugegriffen: 07. Febr. 2018.

Cumming, R. G., Salkeld, G., Thomas, M., \& Szonyi, G. (2000). Prospective study of the impact of fear of falling on activities of daily living, sf-36 scores, and nursing home admission. The Journals of Gerontology Series A: Biological Sciences and Medical Sciences, 55(5), M299-M305.

Dahlbäck, N., Jönsson, A., \& Ahrenberg, L. (1993). Wizard of Oz studies - why and how. Knowledgebased systems, 6(4), 258-266.

Delia, J. G. (1975). Regional dialect, message acceptance, and perceptions of the speaker. Communication Studies, 26(3), 188-194.

Edmonds, W. A., \& Kennedy, T. D. (2016). An applied guide to research designs: Quantitative, qualitative, and mixed methods. Sage verlag.

Epley, N., Waytz, A., \& Cacioppo, J. T. (2007). On seeing human: A three-factor theory of anthropomorphism. Psychological Review, 114(4), 864.

Eyssel, F., Kuchenbrandt, D., Bobinger, S., Ruiter, L. de, \& Hegel, F. (2012). 'If you sound like me, you must be more human': On the interplay of robot and user features on human-robot acceptance and anthropomorphism. In Proceedings of the seventh annual ACM/IEEE internationalconference on Human-Robot Interaction (S. 125-126).

Fischinger, D., Einramhof, P., Papoutsakis, K., Wohlkinger, W., Mayer, P., Panek, P., et al. (2016). Hobbit, a care robot supporting independent living at home: First prototype and lessons learned. Robotics and Autonomous Systems, 75,60-78.

Fischinger, D., Einramhof, P., Wohlkinger, W., Papoutsakis, K., Mayer, P., Panek, P.,et al. (2013). Hobbit - The mutual care robot. In Workshop - Proceedings of ASROB.

Forlizzi, J., DiSalvo, C., \& Gemperle, F. (2004). Assistive robotics and an ecology of elders living independently in their homes. Human-Computer Interaction, 19(1), 25-59.

Frennert, S., Eftring, H., \& Östlund, B. (2017). Case report: Implications of doing research on socially assistive robots in real homes. International Journal of Social Robotics, 1-15. 
Gerling, K., Hebesberger, D., Dondrup, C., Körtner, T., \& Hanheide, M. (2016). Robot deployment in long-term care: Case study on using a mobile robot to support physiotherapy. Zeitschrift für Gerontologie und Geriatrie, 49, 288.

Giuliani, M. V., Scopelliti, M., \& Fornara, F. (2005). Elderly people at home: Technological help in everyday activities. In IEEE International symposium on robot and human interactive communication (ROMAN 2005) (S. 365-370).

Igic, A., Watson, C., Teutenberg, J., Broadbent, E., Tamagawa, R., \& MacDonald, B. (2009). Towards a flexible platform for voice accent and expression selection on a healthcare robot. In Proceedings of the australasian language technology association workshop 2009 (S. 109-113).

Kachouie, R., Sedighadeli, S., Khosla, R., \& Chu, M.-T. (2014). Socially assistive robots in elderly care: A mixed-method systematic literature review. International Journal of Human-Computer Interaction, 30(5), 369-393.

Kidd, C. D., Taggart, W., \& Turkle, S. (2006). A sociable robot to encourage social interaction among the elderly. In Proceedings 2006 IEEE international conference on robotics and automation (ICRA), 2006. (S. 3972-3976).

Klein, B., Graf, B., Schlömer, I. F., Rossberg, H., Röhricht, K., \& Baumgarten, S. (2018). Robotik in der Gesundheitswirtschaft. Einsatzfelder und Potenzial. Medhochzwei.

Mara, M., \& Appel, M. (2015). Effects of lateral head tilt on user perceptions of humanoid and android robots. Computers in Human Behavior, 44, 326-334.

Maxwell, G. M., Cook, M. W., \& Burr, R. (1985). The encoding and decoding of liking from behavioral cues in both auditory and visual channels. Journal of Nonverbal Behavior, 9(4), 239-263.

Nass, C. (2004). Etiquette equality: Exhibitions and expectations of computer politeness. Communications of the ACM, 47(4), 35-37.

Nass, C., Moon, Y., \& Green, N. (1997). Are machines gender neutral? Gender-stereotypic responses to computers with voices. Journal of applied social psychology, 27(10), 864-876.

O'Keefe, D. J. (2002). Persuasion: Theory and research (Bd. 2). Sage.

Pain, H., Gale, C. R., Watson, C., Cox, V., Cooper, C., \& Sayer, A. A. (2007). Readiness of elders to use assistive devices to maintain their independence in the home. Age and Ageing, 36(4), 465-467.

Pineau, J., Montemerlo, M., Pollack, M., Roy, N., \& Thrun, S. (2003). Towards robotic assistants in nursing homes: Challenges and results. Robotics and Autonomous Systems, 42(3), 271-281.

Pollack, M. E., Brown, L., Colbry, D., Orosz, C., Peintner, B., Ramakrishnan, S., et al. (2002). Pearl: A mobile robotic assistant for the elderly. In AAAI workshop on automation as eldercare (Bd. 2002, S. 85-91).

Ramseyer, F., \& Tschacher, W. (2011). Nonverbal synchrony in psychotherapy: Coordinated body movement reflects relationship quality and outcome. Journal of Consulting and Clinical Psychology, 79(3), 284.

Robinson, H., MacDonald, B., Kerse, N., \& Broadbent, E. (2013). The psychosocial effects of a companion robot: A randomized controlled trial. Journal of the American Medical Directors Association, 14(9), 661-667.

Robinson, H., MacDonald, B., \& Broadbent, E. (2014). The role of healthcare robots for older people at home: A review. International Journal of Social Robotics, 6(4), 575-591.

Richardson, R., Marques, P., \& Morgan, K. (2015). Social innovation for an age friendly society.

Scassellati, B. (2000). Investigating models of social development using a humanoid robot.

Scopelliti, M., Giuliani, M. V., \& Fornara, F. (2005). Robots in a domestic setting: A psychological approach. Universal access in the information society, 4(2), 146-155.

Shibata, T. (1999). Seal-type therapeutic robot. http://www.paro.jp/. Zugegriffen: 30. Okt. 2017. 
Stafford, R., MacDonald, B. A., Jayawardena, C., Wegner, D. M., \& Broadbent, E. (2014). Does the robot have a mind? Mind perception and attitudes towards robots predict use of an eldercare robot. International journal of social robotics, 6(1), 17-32.

Syrdal, D. S., Dautenhahn, K., Walters, M. L., \& Koay, K. L. (2008). Sharing spaces with robots in a home scenario-anthropomorphic attributions and their effect on proxemic expectations and evaluations in a live HRI trial. In AAAI Fall Symposium: AI in eldercare: New solutions to old problems (S. 116-123).

Syrdal, D. S., Dautenhahn, K., Woods, S. N., Walters, M. L., \& Koay, K. L. (2007). Looking good? Appearance preferences and robot personality inferences at zero acquaintance. In AAAI Spring Symposium: Multidisciplinary collaboration for socially assistive robotics (S. 86-92).

Takano, E., Matsumoto, Y., Nakamura, Y., Ishiguro, H., \& Sugamoto, K. (2008). Psychological effects of an android bystander on human-human communication. In 8th IEEE-RAS international conference on humanoid robots (2008), (S. 635-639).

Tamagawa, R., Watson, C. I., Kuo, I. H., MacDonald, B. A., \& Broadbent, E. (2011). The effects of synthesized voice accents on user perceptions of robots. International Journal of Social Robotics, 3(3), 253-262.

Terry, G., Hayfield, N., Clarke, V., \& Braun, V. (2017). Thematic analysis. The SAGE Handbook of Qualitative Research in Psychology, 17.

Tidwell, N. D., Eastwick, P. W., \& Finkel, E. J. (2013). Perceived, not actual, similarity predicts initial attraction in a live romantic context: Evidence from the speed-dating paradigm. Personal Relationships, 20(2), 199-215.

Tinetti, M. E., \& Williams, C. S. (1997). Falls, injuries due to falls, and the risk of admission to a nursing home. New England journal of medicine, 337(18), 1279-1284.

Tinker, A., \& Lansley, P. (2005). Introducing assistive technology into the existing homes of older people: Feasibility, acceptability, costs and outcomes. Journal of telemedicine and telecare, 11 (1 suppl), 1-3.

Vandemeulebroucke, T., Casterlé, B. D. de, \& Gastmans, C. (2017). The use of care robots in aged care: A systematic review of argument-based ethics literature. Archives of Gerontology and Geriatrics.

Vincze, M., Fischinger, D., Bajones, M., Wolf, D., Suchi, M., Lammer, L., ... Gisinger, C. (2016). What older adults would like a robot to do in their homes-first results from a user study in the homes of users. In Proceedings of ISR $2016: 47$ st international symposium on robotics (S. 1-7).

Walters, M. L., Koay, K. L., Syrdal, D. S., Dautenhahn, K., \& Te Boekhorst, R. (2009). Preferences and perceptions of robot appearance and embodiment in human-robot interaction trials. Procs of New Frontiers in Human-Robot Interaction.

Walters, M. L., Syrdal, D. S., Dautenhahn, K., Te Boekhorst, R., \& Koay, K. L. (2008). Avoiding the uncanny valley: Robot appearance, personality and consistency of behavior in an attention-seeking home scenario for a robot companion. Autonomous Robots, 24(2), 159-178.

Waytz, A., Cacioppo, J., \& Epley, N. (2010). Who sees human? The stability and importance of individual differences in anthropomorphism. Perspectives on Psychological Science, 5(3), 219-232.

West, T. V., Magee, J. C., Gordon, S. H., \& Gullett, L. (2014). A little similarity goes a long way: The effects of peripheral but self-revealing similarities on improving and sustaining interracial relationships. Journal of personality and social psychology, 107(1), 81.

World Health Organization. (2015). World report on ageing and health. World Health Organization. Wu, Y.-H., Fassert, C., \& Rigaud, A.-S. (2012). Designing robots for the elderly: Appearance issue and beyond. Archives of gerontology and geriatrics, 54(1), 121-126. 
Michael Früh hat einen MSc International Financial Management. Er ist CFO und einer der Inhaber der F\&P Robotics AG. Er ist gelernter Elektroniker, hat Betriebsökonomie und internationales Finanzmanagement studiert und eine Weiterbildung in Gesundheitsökonomie absolviert. Neben seinen Aufgaben als CFO ist Michael Früh insbesondere mit der Entwicklung des Bereiches Pflegerobotik bei F\&P beschäftigt. Dazu gehören der Aufbau von Partnerschaften mit Institutionen im Gesundheitswesen, das Durchführen von Markt- und Bedürfnisanalysen sowie Publikationen und Vorträge an relevanten Veranstaltungen. Vor seinem Engagement bei F\&P Robotics hat Michael Früh vier Jahre als wissenschaftlicher Mitarbeiter am Winterthurer Institut für Gesundheitsökonomie gearbeitet. In dieser Funktion hat er an über 20 Projekten und wissenschaftlichen Studien für Kunden aus dem Gesundheitswesen (Krankenhäuser, Pflegeheime, Pharmaindustrie, Krankenkassen, Kanton) mitgearbeitet. Nebenamtlich ist Michael Früh Mitglied des Verwaltungsrats der sensiQoL AG, einer Firma, welche sich auf die Lebensqualität von Menschen in einem Betreuungsverhältnis spezialisiert hat. Er ist Gastdozent an der Zürcher Hochschule für Angewandte Wissenschaften (ZHAW) und Mitautor von mehreren Artikeln und Büchern zu den Themen Robotik im Gesundheitswesen und Lebensqualität. Zudem ist er Finanzvorstand von FamilyStart Zürich, einem gemeinnützigen Verein, der sich für eine optimale nachgeburtliche Versorgung von Mutter und Kind einsetzt.

Alina Gasser hat einen Master of Science in Psychologie. Sie absolvierte verschiedene Praktika im Spital- und Rehabereich und arbeitete kurze Zeit als Pflegehilfe. Während des Studiums arbeitete sie am Institut für Bildungsevaluation und war dort zwei Jahre lang für die Administration und Projektorganisation zuständig. Seit Abschluss des Masters in Psychologie mit Schwerpunkt Human-Computer Interaction an der Universität Basel arbeitet sie hauptsächlich bei ergonomie \& technologie (e\&t) $\mathrm{GmbH}$ als Usability Researcher. Sie ist zuständig für die Gestaltung, Ausführung, Analyse und das Reporting für benutzerzentriertes Design Research und Usability-Tests und mitverantwortlich für das Erstellen von Prototypen, User-Flows sowie Konzeptentwicklung für neue und bestehende Produkte.

Open Access Dieses Kapitel wird unter der Creative Commons Namensnennung 4.0 International Lizenz (http://creativecommons.org/licenses/by/4.0/deed.de) veröffentlicht, welche die Nutzung, Vervielfältigung, Bearbeitung, Verbreitung und Wiedergabe in jeglichem Medium und Format erlaubt, sofern Sie den/die ursprünglichen Autor(en) und die Quelle ordnungsgemäß nennen, einen Link zur Creative Commons Lizenz beifügen und angeben, ob Änderungen vorgenommen wurden.

Die in diesem Kapitel enthaltenen Bilder und sonstiges Drittmaterial unterliegen ebenfalls der genannten Creative Commons Lizenz, sofern sich aus der Abbildungslegende nichts anderes ergibt. Sofern das betreffende Material nicht unter der genannten Creative Commons Lizenz steht und die betreffende Handlung nicht nach gesetzlichen Vorschriften erlaubt ist, ist für die oben aufgeführten Weiterverwendungen des Materials die Einwilligung des jeweiligen Rechteinhabers einzuholen.

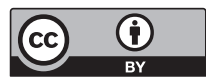

